\title{
The impact of diabetes mellitus on drug resistance in patients with newly diagnosed tuberculosis: a systematic review and meta- analysis
}

\author{
Dong Huang ${ }^{1}$, Yan Wang ${ }^{2}$, Yu Wang ${ }^{1}$, Zongan Liang ${ }^{1}$ \\ ${ }^{1}$ Department of Respiratory and Critical Care Medicine, ${ }^{2}$ Department of Thoracic Surgery, West China Hospital, Sichuan University, Chengdu \\ 610041, China \\ Contributions: (I) Conception and design: D Huang, Y Wang, Y Wang, Z Liang; (II) Administrative support: Z Liang; (III) Provision of study \\ materials or patients: D Huang, Y Wang, Y Wang; (IV) Collection and assembly of data: D Huang, Y Wang, Y Wang; (V) Data analysis and \\ interpretation: D Huang, Y Wang; (VI) Manuscript writing: All authors; (VII) Final approval of manuscript: All authors. \\ \#These authors contributed equally to this work. \\ Correspondence to: Zongan Liang. Department of Respiratory and Critical Care Medicine, West China Hospital, Sichuan University. No.37 Guoxue \\ Alley, Chengdu, 610041, China. Email: liangza@scu.edu.cn.
}

\begin{abstract}
Background: Tuberculosis and diabetes mellitus are both important global health problems now. Previous studies have drawn different conclusions about the impact of diabetes on drug resistance in patients with newly diagnosed tuberculosis.

Methods: We conducted a systematic search in four databases: PubMed, EMBSE, Cochrane Library, and Web of Science. The relative risk (RR) was applied to assess the association of diabetes with drug resistance and the STATA version 12.0 was used for data synthesis.

Results: A total of 13 studies involving 33,747 patients were included in our study. The pooled results revealed that presence of diabetes was significantly associated with isoniazid resistance ( $\mathrm{RR}=1.22,95 \% \mathrm{CI}$ : 1.04-1.43) in patients with newly diagnosed tuberculosis. However, no significant impact of diabetes on rifampicin resistance $(\mathrm{RR}=0.67,95 \% \mathrm{CI}: 0.41-1.11)$ or multi-drug resistance (MDR) $(\mathrm{RR}=1.28,95 \% \mathrm{CI}$ : 0.93-1.75) was observed. The results of subgroup analysis were similar to the pooled results. No significant publication bias for the results of MDR was found.

Conclusions: In patients with newly diagnosed tuberculosis, diabetes is associated with isoniazid resistance. However, there is no significant impact of diabetes on the rifampicin resistance or MDR. However, these findings still need to be verified in the future.
\end{abstract}

Keywords: Tuberculosis (TB); diabetes mellitus; drug resistance; meta-analysis

Submitted Aug 16, 2019. Accepted for publication Jan 03, 2020.

doi: 10.21037/apm.2020.02.16

View this article at: http://dx.doi.org/10.21037/apm.2020.02.16

\section{Introduction}

There were 10.4 million new cases of tuberculosis (TB) and 1.7 million deaths from tuberculosis globally in 2016 (1). Although this number has decreased dramatically in the last few decades, tuberculosis is still the top cause of death among infectious diseases now and a great challenge to global health security (2). Some risk factors are associated with tuberculosis infection or latent tuberculosis reactivation, such as smoking, alcohol intake, poor living conditions, malnutrition, contacts with sputum smear positive patients, HIV, kidney dialysis, organ transplantation, etc. $(3,4)$. On the other hand, lack of systems and tools to monitor and treat TB patients in some high-incidence countries and international migration flows might both increase the global TB incidence rate $(5,6)$. Drug-resistant tuberculosis (DR-TB) has generated lots of 
concerns and discussion over recent years. In 2006, about 600,000 new cases were resistant to rifampicin, the most powerful and commonly used first-line drug (1). About $4.1 \%$ of new tuberculosis cases and $19 \%$ of retreatment cases experience multidrug-resistant tuberculosis (MDR-TB) globally now (7). It is generally recognized that the genetic selection of drug resistance strains makes mycobacterium tuberculosis (M. tuberculosis) unresponsive to drugs (8). Some man-related factors such as misdiagnosis and mismanagement could also contribute to this problem (9). It is noticeable that DR-TB patients generally require longer treatment regimens and more costly, toxic secondline drugs, which might cause more complications (10).

Diabetes mellitus is also one of the most important global health problems now (11). The diabetes prevalence, health expenditure and deaths due to diabetes would continue to rise in the next decades (12). One of the biggest threats caused by diabetes is the complications. Microvascular complications, macrovascular complications, as well as miscellaneous complications affect nearly every organ system (13).

Some previous studies have revealed that DR-TB might be associated with diabetes. For example, some researchers found there was a significant correlation between diabetes and DR -TB or MDR-TB development and adverse clinical outcomes $(14,15)$. The renal lesions, effects of blood glucose on plasma concentration of antituberculosis drugs, influences of reactive oxygen species (ROS) on drug resistance mutations (16) are all potential mechanisms. However, some other studies showed opposite results $(17,18)$. The differences of population, history of tuberculosis and treatment strategy might cause the inconsistency of conclusions. Thus, this issue is worth exploring further.

Given the uncertain relationship between diabetes mellitus and DR-TB, we conducted this systematic review and meta-analysis to clarify the impact of diabetes mellitus on drug resistance in patients with newly diagnosed tuberculosis.

\section{Methods}

\section{Search strategy}

We conducted a systematic search in four databases: PubMed, EMBASE, Cochrane Library, and Web of Science. We aimed to identify all potentially related studies which were published from January 1, 1966 to July 1, 2019.
We used both $\mathrm{MeSH}$ terms and free-text words to increase sensitivity.

The following search terms were used: "diabetes" or "diabetes mellitus" or "diabetics mellitus" or "diabetic" or "diabete" and "tuberculosis" or "tuberculoses" or "tuberculosis" or "pulmonary tuberculosis" and "drug resistance" or "drug-resistance" or "drug resistant" or "DR".

All searched results were evaluated according to the Preferred Reporting Items for Systematic Reviews and Meta-Analyses (PRISMA) statement (19). Firstly, titles and abstracts were screened to recognize relevant studies. Then, full texts were evaluated carefully. Both search and selection were completed by two independent investigators (Dong Huang and Yan Wang).

\section{Inclusion and exclusion criteria}

The following inclusion criteria were used: (I) adults (18 years or older); (II) newly diagnosis of tuberculosis with or without diabetes mellitus; (III) received antituberculotic therapy and follow-up; (IV) outcomes included but not limited to: DR [M. tuberculosis resistant to any first-line anti-TB drug (isoniazid, rifampicin, pyrazinamide and ethambutol)] or MDR (M. tuberculosis resistant to at least isoniazid and rifampin).

The following exclusion criteria were used: (I) previous history of TB, TB treatment, DR-TB or MDR-TB; (II) editorials, expert opinions, case reports and reviews or studies with insufficient data; (III) nonhuman studies.

\section{Data collection}

Two researchers (Dong Huang and Yan Wang) collected data from each article by using an Excel sheet (Microsoft Corporation) independently. Any disagreement was resolved through team discussion until consensus reached. The following information was extracted from all included studies: first author of the study, publication year, the country where the study was performed, sample size, number of diabetes patients, type of diabetes, number of DR, number of MDR, type of drug-resistance.

\section{Statistical analyses}

Data synthesis was accomplished by STATA (version 12.0; Stata Corporation) and the results were displayed by forest plots. We rendered statistical significance as $\mathrm{P}$ 


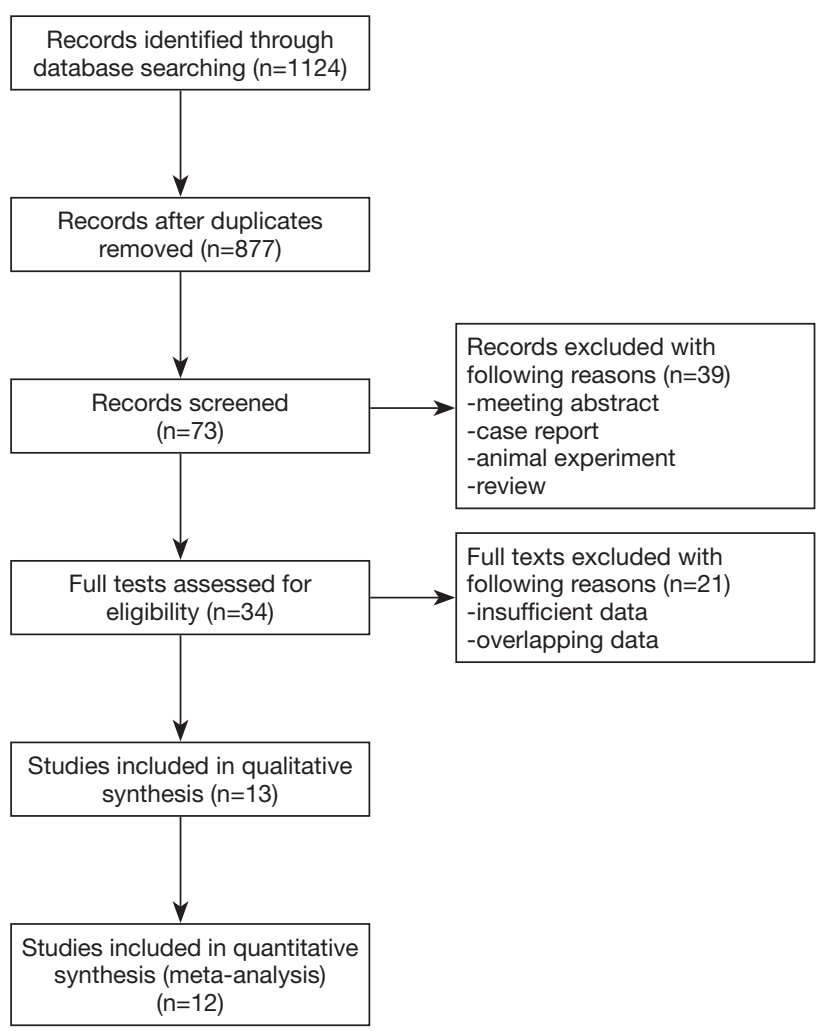

Figure 1 The flow diagram of this meta-analysis.

value $<0.05$. Statistical heterogeneity between studies was evaluated using Cochran's Q test and Higgins $\mathrm{I}^{2}$ statistic (20). The significant heterogeneity was defined as $\mathrm{P}<0.1$ and (or) $\mathrm{I}^{2}>50 \%$. We used random-effects model to calculate the pooled effects if significant heterogeneity was observed, or used the fixed-effects model when there was no significant heterogeneity. We calculated mean difference (MD) and 95\% confidence interval (CI) for continuous data, while calculated risk ratio (RR) and $95 \%$ CI for dichotomous data. We also performed a subgroup analysis investigating whether country, sample size, type of drugresistance, location of tuberculosis affected results of our study. We performed the sensitivity analysis to further verify conclusions of our study by removing the data of an individual study in turn each time. We evaluated publication bias by the Begg's funnel plot and Egger's linear regression tests (21).

\section{Quality assessment}

The quality of the included studies was assessed with the NOS (Newcastle-Ottawa quality assessment scale) (22). The
NOS included three parameters: selection, comparability, and outcome. Studies which earned at least 6 points were regarded as high-quality studies. Quality assessment was also completed by two independent researchers (Dong Huang and Yan Wang).

\section{Results}

\section{Characteristics of included studies}

As shown in Figure 1, the electronic search yielded 1,124 records. After duplicates were removed, there were 877 records left. Then we screened titles and abstracts carefully, and assessed 34 full-text articles for eligibility. At last, 13 articles (17,18,23-33) including 33,747 patients were included in this meta-analysis, among which 6,365 patients suffered from diabetes. In these 13 studies, 959 patients were found with isoniazid resistance, and 188 patients were found with rifampicin resistance, and only one patient were reported resistant to ethambutol. Moreover, a total of 641 patients had MDR-TB. There were 8 studies which focused on pulmonary tuberculosis exclusively. Only some of included studies reported the type of diabetes and type of drug resistance. As for quality assessment, all studies earned at least 6 points. Other details of characteristics of included studies were shown in Table 1.

\section{Impact of diabetes on drug resistance of tuberculosis}

There were 5 studies which explored the impact of diabetes on isoniazid resistance. The fixed-effects model was applied because no significant heterogeneity was observed $\left(\mathrm{I}^{2}=0 \%\right.$, $\mathrm{P}=0.471)$. The pooled results revealed a significant trend towards more resistance to isoniazid in diabetes patients compared with non-diabetes patients ( $\mathrm{RR}=1.22,95 \% \mathrm{CI}$ : 1.04-1.43) (Figure 2).

There were 5 studies which explored the impact of diabetes on rifampicin resistance. At last 3 studies were included in our meta-analysis because the data of other 2 studies could not be used to calculate the RR in STATA. We used fixed-effects model because of the non-significant heterogeneity ( $\mathrm{I}^{2}=0 \%, \mathrm{P}=0.712$ ). There was no significant impact of diabetes on rifampicin resistance ( $\mathrm{RR}=0.67,95 \%$ CI: 0.41-1.11) (Figure 3).

Totally, 13 studies reported the association between diabetes and MDR-TB. Similarly, 10 studies with valid data were included in our meta-analysis. The random-effects model was applied because of significant heterogeneity 
Table 1 Basic characteristics of included studies

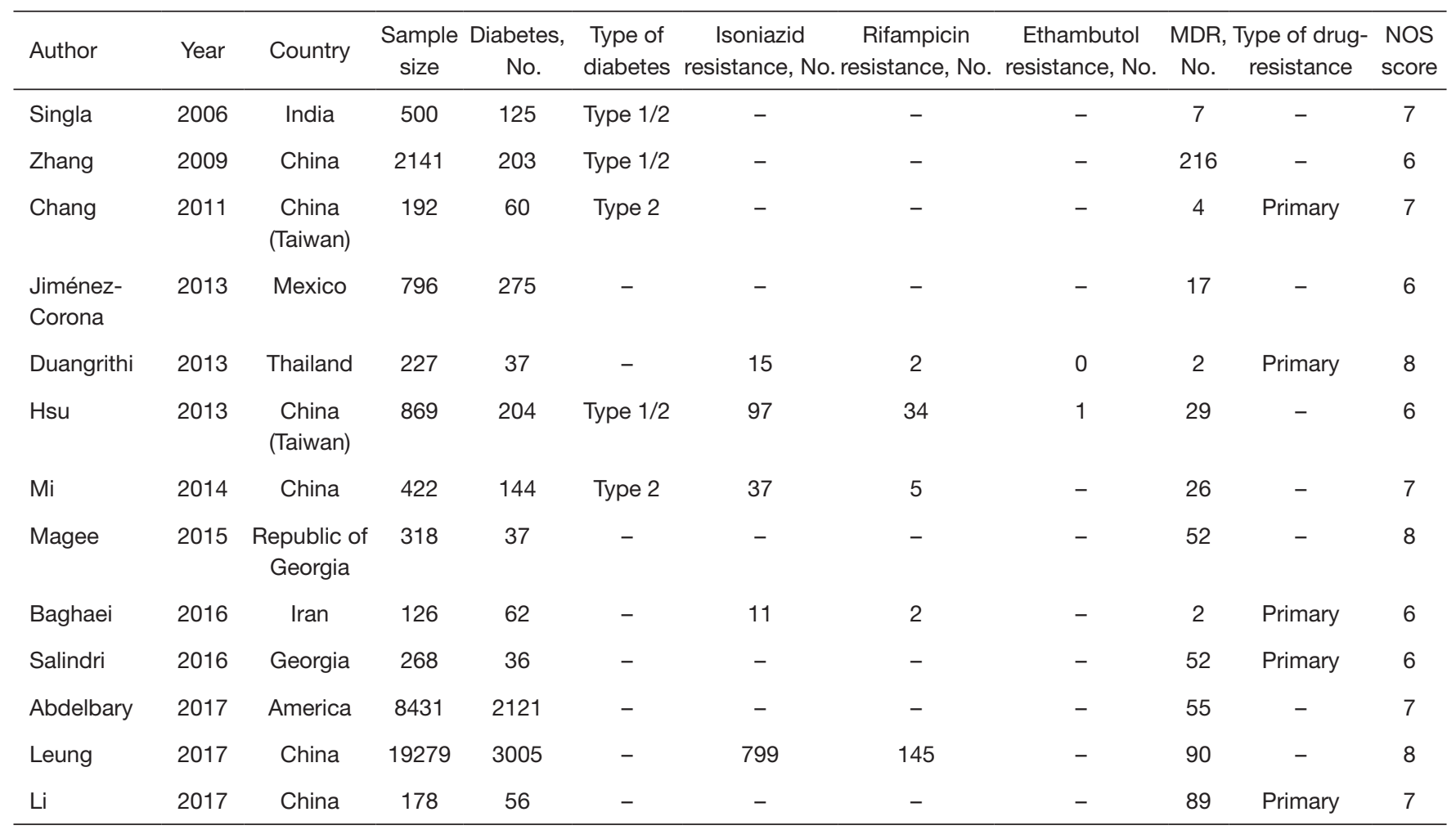

MDR, multidrug resistance; NOS, Newcastle-Ottawa quality assessment scale; -, not reported; No., number.

Study

ID

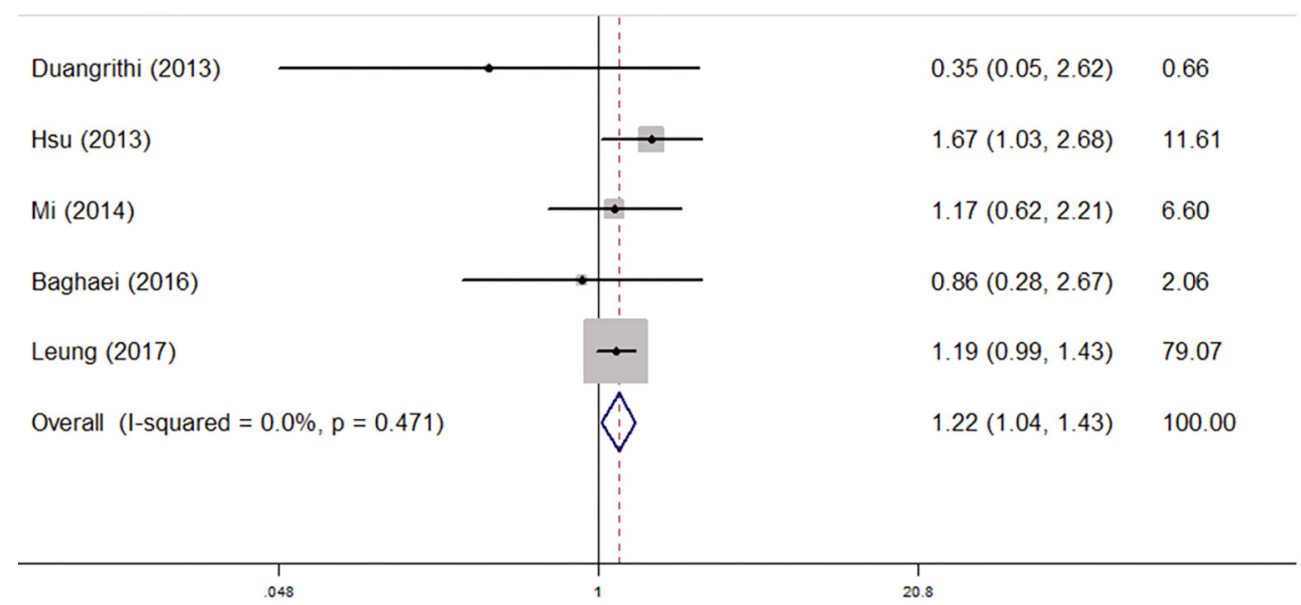

Figure 2 Forest plot for the association between diabetes and isoniazid resistance in newly diagnosed tuberculosis patients. 


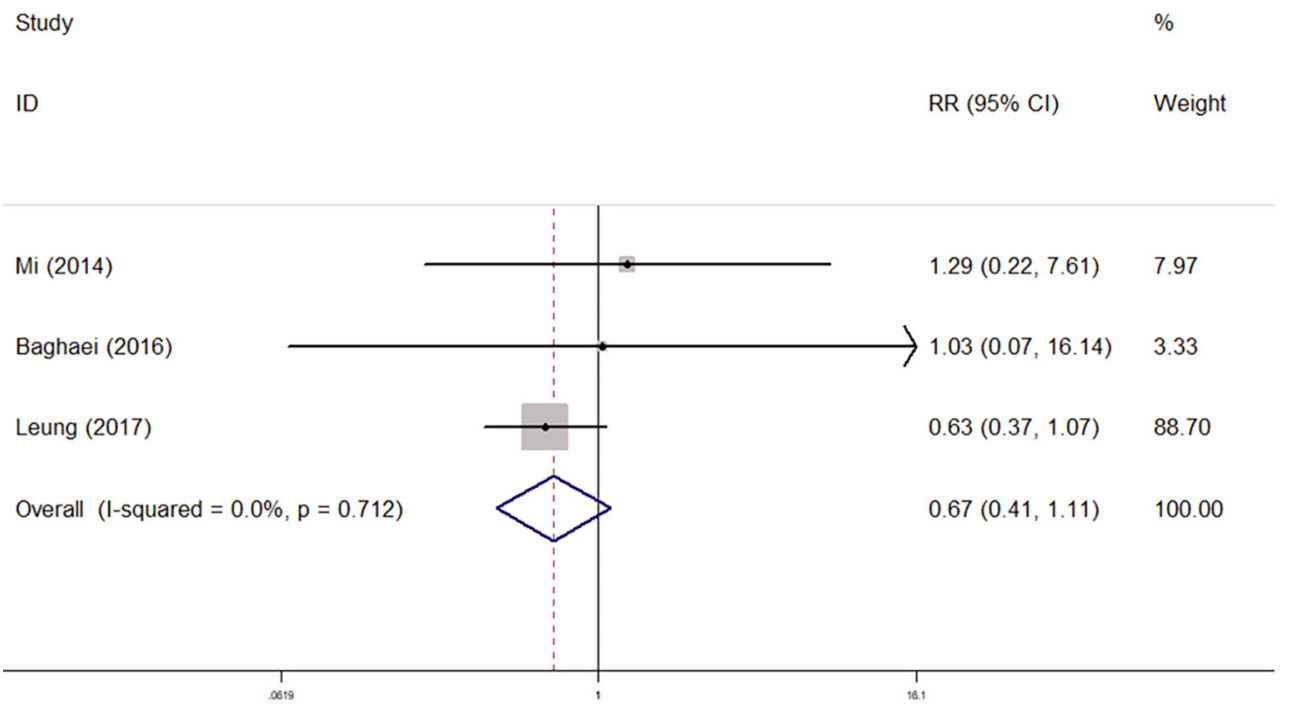

Figure 3 Forest plot for the association between diabetes and rifampicin resistance in newly diagnosed tuberculosis patients.

Study
ID

Figure 4 Forest plot for the association between diabetes and multi-drug resistance (MDR) in newly diagnosed tuberculosis patients.

$\left(\mathrm{I}^{2}=62.2 \%, \mathrm{P}=0.005\right)$. The pooled results showed that diabetes was not associated with $\mathrm{MDR}(\mathrm{RR}=1.28,95 \% \mathrm{CI}$ : 0.93-1.75) (Figure 4).

\section{Subgroup analysis}

We aimed to investigate whether country (China vs non-
China studies), sample size $(\geq 1,000$ vs $<1,000)$, type of drug-resistance (primary drug-resistance) and location of tuberculosis (pulmonary tuberculosis) would affect the results of MDR-TB. Most of the results of subgroup analysis were similar to pooled results. The sample size, type of drug-resistance and location of tuberculosis did not have significant effects on the association between 
Table 2 Meta-analyses for the association of diabetes mellitus with MDR in patients with newly diagnosed tuberculosis

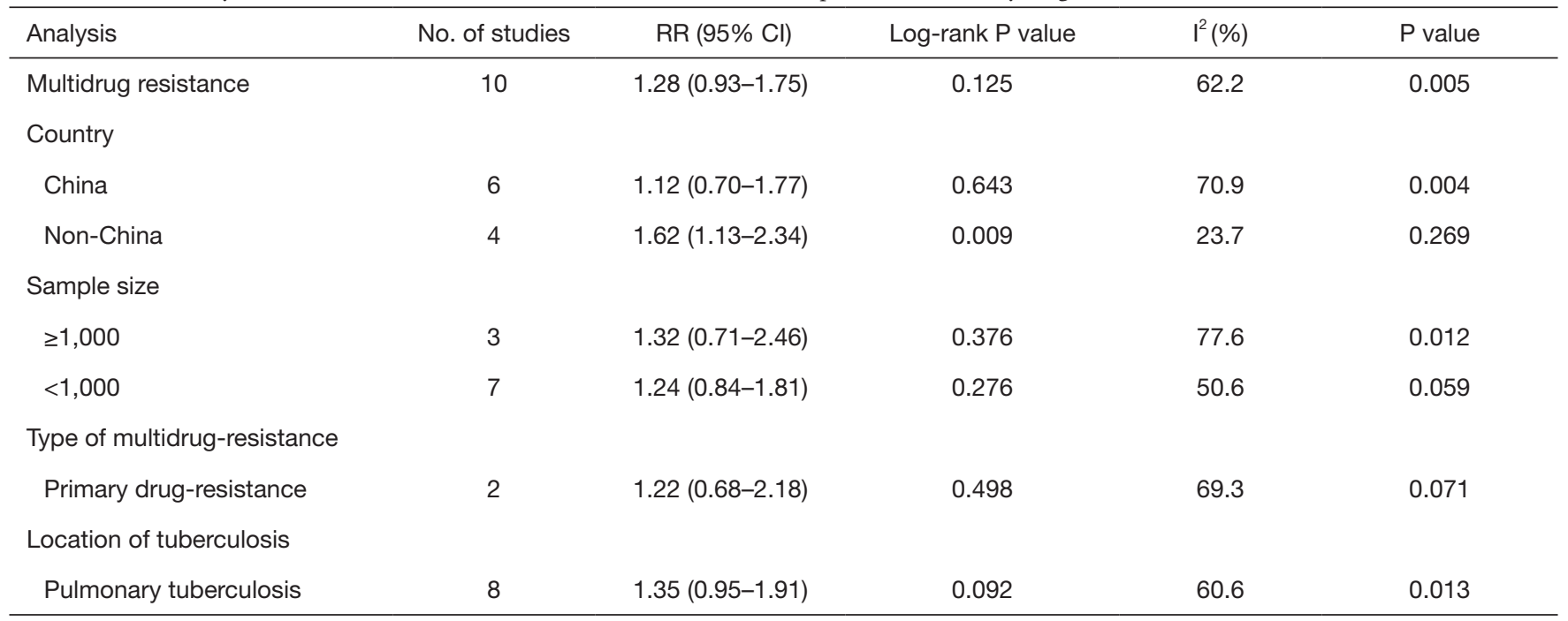

MDR, multidrug resistance; No., number; RR, relative risk; $\mathrm{Cl}$, confidence interval.

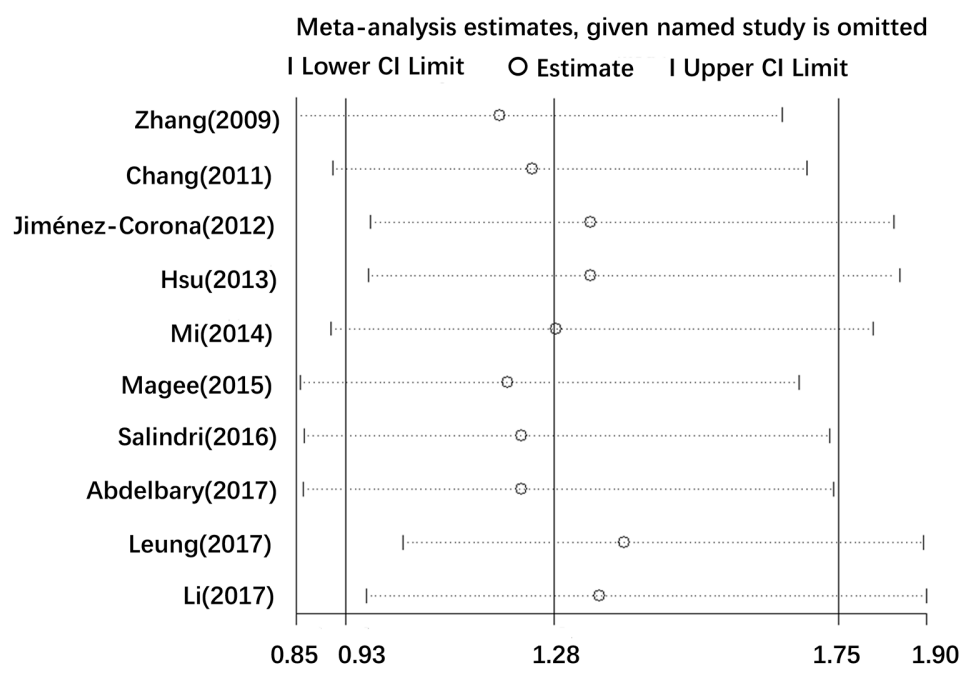

Figure 5 Sensitivity analysis for the results of multi-drug resistance.

diabetes and MDR-TB. However, the pooled results of 4 non-China studies showed diabetes could increase the risk of MDR-TB ( $\mathrm{RR}=1.62,95 \% \mathrm{CI}: 1.13-2.34)$ (Table 2).

\section{Sensitivity analysis and publication bias}

Because of the significant heterogeneity in the pooled results of MDR, we further conducted sensitivity analysis to assess the stability of the pooled results. We evaluated the exact influences of each study on the pooled RR by excluding every single study individually and gradually from the overall meta-analysis. The results showed that the pooled RR for the impact of diabetes on MDR were robust (Figure 5).

The publication bias was evaluated by Begg's funnel plot and Egger's linear regression tests. The funnel plot was symmetric and we did not observe significant publication bias for the results of MDR (Begg's: $\mathrm{P}=0.859$; Egger's: $\mathrm{P}=0.771$ ) (Figure 6). 


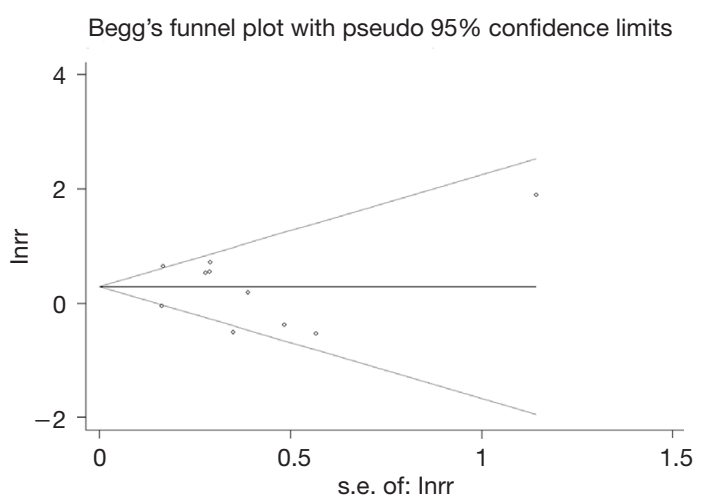

Figure 6 Publication bias for the results of multi-drug resistance.

\section{Discussion}

As far as we know, this is the first systemic review and metaanalysis investigating exclusively the impact of diabetes on drug resistance in newly diagnosed tuberculosis patients. We found that, compared with absence of diabetes, presence of diabetes was associated with resistance to isoniazid in newly diagnosed tuberculosis patients. However, diabetes did not have significant impact on the resistance to rifampicin or MDR.

We supposed that there might be several reasons why diabetes had significant effects on isoniazid rather than rifampicin. Firstly, the number of studies included in the meta-analysis for rifampicin resistance is smaller than that for isoniazid resistance. The pooled results might be insufficient to find a link between diabetes and rifampicin resistance. Secondly, isoniazid is one of the first known and most effective anti-TB drugs (34). It has strong early bactericidal activity and is long-term widely used. Numerous researchers believe resistance to isoniazid usually comes first during first-line chemotherapy regimens among new patients without HIV-infected in most settings (35-37). On the other hand, isoniazid resistance is even considered as the first step in development of MDR and extremely resistant (XDR) TB (38). Thirdly, the serum concentrations of isoniazid and rifampin are often affected by blood glucose level and become below the expected range $(39,40)$. Furthermore, tuberculosis and the drugs used to treat tuberculosis might also lead to worse glycemic control in people with diabetes (41). Tuberculosis and diabetes might aggravate each other and this vicious circle could produce the effects similar to misadministration of anti-TB drugs, which is the most common reason for drug resistance (42). Heysell et al. have found the dosage adjustment of rifampin was more likely to elevate serum concentration to the target range than dosage adjustment of isoniazid (43), which might partly explain why isoniazid resistance is more common in patients with TB and diabetes. Moreover, the changed binding drugs in the blood due to glycosylation of plasma proteins and increased renal clearance of antiTB drugs have both been reported in previous studies (44). However, these theories have not been be confirmed widely and the scientific mechanism is still unclear. The relationship between diabetes and DR-TB is worth discussing in the future. In addition to this, we have planned to explore if diabetes would increase the risks of resistance to pyrazinamide and ethambutol. Nevertheless, only 2 researches reported the incidence of ethambutol resistance. We abandoned this idea due to lack of data.

Compared with single drug-resistance, there were more researches which explored the association between diabetes and MDR. Liu et al. have conducted a meta-analysis of 13 studies and concluded that diabetes was an independent risk factor for MDR-TB (OR =1.71; 95\% CI: 1.32-2.22) (45). However, their meta-analysis included both newly diagnosed patients and retreatment patients with history of TB. In their meta-analysis, only about half of included studies focused on newly diagnosed patients exclusively. We thought that it was necessary to analyze new patients separately because it has been reported that previously treated patients were at increased risk for drug resistance (46). Tsao et al. have also found that a history of pulmonary TB was a predictor for drug resistance (47). Furthermore, the emergence of MDR-TB is often related to abuse or nonstandard use of anti-TB drugs, which mainly happens in retreatment patients with long-term medication. Tegegne et al. have also conducted a similar meta-analysis about the association between diabetes and MDR-TB (48). In their particular sub-analysis including only 5 studies of new TB, the pooled OR (95\% CI) for MDR were 2.36 (1.59-3.51) when adjusted for at least one covariate and 1.64 (0.99-2.71) when not adjusted for covariates. The authors also deemed that there were inadequate number of studies which adjusted and adjusted results still suffered from unobserved confounding factors. This controversial conclusion needs to be clarified in future multi-center, large-scale and welldesigned prospective studies. As for the results of subgroup analysis of non-China studies, we should be cautious about this conclusion. As reported the number of TB in China is 900,000 in 2016, only less than India and Indonesia (49). However, after consulting relative references, we did not find distinct differences of population, treatments or follow- 
up systems between China and other countries. It needs to be further explored. To sum up, we believe the current evidence is not enough to certify the association between diabetes and MDR in newly diagnosed TB patients. Physicians should pay more attention to the level of blood glucose in patients with history of $\mathrm{TB}$ or retreatment patients specially to minimize the risks of MDR-TB. This measure would decrease mutual impact of diabetes and tuberculosis (50) and is cost-effective for these patients (51).

Apart from the direct effects of diabetes on drug resistance, now evidence is growing that diabetes could affect the occurrence and prognosis of tuberculosis. Previous studies have ever found that diabetic patients have increased risk of developing TB compared with people without DM $(52,53)$. Moreover, the risks of developing $\mathrm{TB}$ are especially high in young, low BMI, and poorly controlled diabetes patients (54). Diabetes might cause immune dysfunction, altered levels of cytokines and changed activation state of the macrophages $(55,56)$. Meanwhile, the acid environment of high glucose may be in favor of growth of $M$. tuberculosis. This mechanism would decrease efficacy of anti-TB drugs and increase the risks of drug resistance. Baker et al. have conducted a systemic review and revealed diabetes increased the risk of treatment failure, relapse and death among TB patients (57). Meanwhile, the time of sputum culture conversion is longer in diabetes patients compared with non- diabetes patients (58). Recently oxidative stress is also considered as a major underlying mechanism adversely impacting TB treatment outcomes of diabetes patients (59). However, these findings have not been confirmed in other studies. It is reported that diabetes is not associated with higher OR of unsuccessful treatment outcome in MDR/XDRTB patients (60). Some researchers even found diabetes appeared as a protective factor for death in TB patients (61). Future studies should obtain more information about the characteristics of population, comorbidities (especially HIV infection and kidney diseases) and treatments in order to draw relatively fair conclusions.

The effects of glycemic control conditions and types of diabetes on TB is also a matter of concern. Previous studies got different results. Shewade HD once conducted a systemic review and concluded that optimal glycemic control was associated with more successful treatment outcomes, fewer odds of recurrence, faster culture conversion (62). As for the types of diabetes, it was reported that the blood concentration of drugs and therapy outcomes of TB were more favorable in patients with type 1 diabetes mellitus compared with type 2 (63). Researchers speculated that the significant slower inactivation process and slighter inactivation of anti-TB drugs in type 1 diabetes patients were main reasons. We have also planned to explore whether glycemic control conditions and diabetes type could influence drug resistance. Similarly, we abandoned this idea because lack of available data in included studies. However, we believe it is worth exploring in the future. After understanding more specific relationships between diabetes and $\mathrm{TB}$, physicians could make more detailed treatment plans, which might lead to better prognosis for TB patients with diabetes.

There are still several limitations in the study. Firstly, significant heterogeneity exists in this meta-analysis. But we did not find any single study which could increase heterogeneity prominently in sensitivity analysis. Then, the included patients in these studies has different types and therapy methods of diabetes. However, we were not able to perform more subgroup analyses or adjust results in terms of these confounding factors due to lack of original data. Thirdly, all studies were published in English in our metaanalysis, which means that some eligible studies might have been excluded because of language restrictions.

\section{Conclusions}

In newly diagnosed tuberculosis patients, presence of diabetes is associated with more resistance to isoniazid compared with absence of diabetes. However, there is no significant impact of diabetes on the rifampicin resistance or MDR. These findings need to be confirmed in the future.

\section{Acknowledgments}

Funding: This work was partially supported by National Science and Technology Major Project of China (2018ZX10715003).

\section{Footnote}

Conflicts of Interest: The authors have no conflicts of interest to declare.

Ethical Statement: The authors are accountable for all aspects of the work in ensuring that questions related to the accuracy or integrity of any part of the work are appropriately investigated and resolved. 
Open Access Statement: This is an Open Access article distributed in accordance with the Creative Commons Attribution-NonCommercial-NoDerivs 4.0 International License (CC BY-NC-ND 4.0), which permits the noncommercial replication and distribution of the article with the strict proviso that no changes or edits are made and the original work is properly cited (including links to both the formal publication through the relevant DOI and the license). See: https://creativecommons.org/licenses/by-nc-nd/4.0/.

\section{References}

1. Floyd K, Glaziou P, Zumla A, et al. The global tuberculosis epidemic and progress in care, prevention, and research: an overview in year 3 of the End TB era. Lancet Respir Med 2018;6:299-314.

2. Glaziou P, Floyd K, Raviglione MC. Global Epidemiology of Tuberculosis. Semin Respir Crit Care Med 2018;39:271-85.

3. Ai JW, Ruan QL, Liu QH, et al. Updates on the risk factors for latent tuberculosis reactivation and their managements. Emerg Microbes Infect 2016;5:e10.

4. Melsew YA, Doan TN, Gambhir M, et al. Risk factors for infectiousness of patients with tuberculosis: a systematic review and meta-analysis. Epidemiol Infect 2018;146:345-53.

5. Uplekar M, Atre S, Wells WA, et al. Mandatory tuberculosis case notification in high tuberculosisincidence countries: policy and practice. Eur Respir J 2016;48:1571-81.

6. Sánchez-Montalvá A, Salvador F, Molina-Morant D, et al. Tuberculosis and immigration. Enferm Infecc Microbiol Clin 2018;36:446-55.

7. Daley CL, Caminero JA. Management of MultidrugResistant Tuberculosis. Semin Respir Crit Care Med 2018;39:310-24.

8. Talukdar D, Sharma R, Sharma AK, et al. Drug resistance in tuberculosis: how to counter the menace? Curr Pharm Biotechnol 2014;15:1158-65.

9. Poce G, Biava M. Overcoming drug resistance for tuberculosis. Future Microbiol 2015;10:1735-41.

10. Falzon D, Schunemann HJ, Harausz E, et al. World Health Organization treatment guidelines for drugresistant tuberculosis, 2016 update. Eur Respir J 2017;49. doi: 10.1183/13993003.02308-2016.

11. Zimmet P, Alberti KG, Magliano DJ, et al. Diabetes mellitus statistics on prevalence and mortality: facts and fallacies. Nat Rev Endocrinol 2016;12:616-22.
12. Ogurtsova K, da Rocha Fernandes JD, Huang Y, et al. IDF Diabetes Atlas: Global estimates for the prevalence of diabetes for 2015 and 2040. Diabetes Res Clin Pract 2017;128:40-50.

13. Papatheodorou K, Papanas N, Banach M, et al. Complications of Diabetes 2016. J Diabetes Res 2016;2016:6989453.

14. Saktiawati AMI, Subronto YW. Influence of Diabetes Mellitus on the Development of Multi Drug ResistantTuberculosis in Yogyakarta. Acta Med Indones 2018;50:11-7.

15. Perez-Navarro LM, Restrepo BI, Fuentes-Dominguez FJ, et al. The effect size of type 2 diabetes mellitus on tuberculosis drug resistance and adverse treatment outcomes. Tuberculosis (Edinb) 2017;103:83-91.

16. Gagneux S, Burgos MV, DeRiemer K, et al. Impact of bacterial genetics on the transmission of isoniazid-resistant Mycobacterium tuberculosis. PLoS Pathog 2006;2:e61.

17. Leung CC, Yew WW, Mok TYW, et al. Effects of diabetes mellitus on the clinical presentation and treatment response in tuberculosis. Respirology 2017;22:1225-32.

18. Mi F, Jiang G, Du J, et al. Is resistance to anti-tuberculosis drugs associated with type 2 diabetes mellitus? A register review in Beijing, China. Glob Health Action 2014;7:24022.

19. Moher D, Liberati A, Tetzlaff J, et al. Preferred reporting items for systematic reviews and meta-analyses: the PRISMA statement. BMJ 2009;339:b2535.

20. Higgins JP, Thompson SG. Quantifying heterogeneity in a meta-analysis. Stat Med 2002;21:1539-58.

21. Begg CB, Mazumdar M. Operating characteristics of a rank correlation test for publication bias. Biometrics 1994;50:1088-101.

22. Stang A. Critical evaluation of the Newcastle-Ottawa scale for the assessment of the quality of nonrandomized studies in meta-analyses. Eur J Epidemiol 2010;25:603-5.

23. Abdelbary BE, Garcia-Viveros M, Ramirez-Oropesa H, et al. Predicting treatment failure, death and drug resistance using a computed risk score among newly diagnosed TB patients in Tamaulipas, Mexico. Epidemiol Infect 2017;145:3020-34.

24. Baghaei P, Tabarsi P, Javanmard P, et al. Impact of diabetes mellitus on tuberculosis drug resistance in new cases of tuberculosis. J Glob Antimicrob Resist 2016;4:1-4.

25. Chang JT, Dou HY, Yen CL, et al. Effect of Type 2 Diabetes Mellitus on the Clinical Severity and Treatment Outcome in Patients With Pulmonary Tuberculosis: A Potential Role in the Emergence of Multidrug-resistance. 
J Formos Med Assoc 2011;110:372-81.

26. Duangrithi D, Thanachartwet V, Desakorn V, et al. Impact of diabetes mellitus on clinical parameters and treatment outcomes of newly diagnosed pulmonary tuberculosis patients in Thailand. Int J Clin Pract 2013;67:1199-209.

27. Hsu AH, Lee JJ, Chiang CY, et al. Diabetes is associated with drug-resistant tuberculosis in Eastern Taiwan. Int J Tuberc Lung Dis 2013;17:354-6.

28. Jiménez-Corona ME, Cruz-Hervert LP, Garcia-Garcia $\mathrm{L}$, et al. Association of diabetes and tuberculosis: Impact on treatment and post-treatment outcomes. Thorax 2013;68:214-20.

29. Li D, He W, Chen B, et al. Primary multidrug-resistant tuberculosis versus drug-sensitive tuberculosis in nonHIV-infected patients: Comparisons of CT findings. PLoS One 2017;12:e0176354.

30. Magee MJ, Kempker RR, Kipiani M, et al. Diabetes mellitus is associated with cavities, smear grade, and multidrug-resistant tuberculosis in Georgia. Int J Tuberc Lung Dis 2015;19:685-92.

31. Salindri AD, Kipiani M, Kempker RR, et al. Diabetes reduces the rate of sputum culture conversion in patients with newly diagnosed multidrug-resistant tuberculosis. Open Forum Infect Dis 2016;3:ofw126.

32. Singla R, Khan N, Al-Sharif N, et al. Influence of diabetes on manifestations and treatment outcome of pulmonary TB patients. Int J Tuberc Lung Dis 2006;10:74-9.

33. Zhang Q, Xiao H, Sugawara I. Tuberculosis Complicated by Diabetes Mellitus at Shanghai Pulmonary Hospital, China. Jpn J Infect Dis 2009;62:390-1.

34. Hu YQ, Zhang $\mathrm{S}$, Zhao F, et al. Isoniazid derivatives and their anti-tubercular activity. Eur J Med Chem 2017;133:255-67.

35. Chiang CY, Centis R, Migliori GB. Drug-resistant tuberculosis: past, present, future. Respirology 2010;15:413-32.

36. Yoshiyama T, Yanai H, Rhiengtong D, et al. Development of acquired drug resistance in recurrent tuberculosis patients with various previous treatment outcomes. Int $\mathrm{J}$ Tuberc Lung Dis 2004;8:31-8.

37. Garg K, Saini V, Dhillon R, et al. Isoniazid mono-resistant tuberculosis: Time to take it seriously. Indian J Tuberc 2019;66:247-52.

38. Nagel S, Streicher EM, Klopper M, et al. Isoniazid Resistance and Dosage as Treatment for Patients with Tuberculosis. Curr Drug Metab 2017;18:1030-9.

39. Reynolds J, Heysell SK. Understanding pharmacokinetics to improve tuberculosis treatment outcome. Expert Opin
Drug Metab Toxicol 2014;10:813-23.

40. Heysell SK, Moore JL, Staley D, et al. Early Therapeutic Drug Monitoring for Isoniazid and Rifampin among Diabetics with Newly Diagnosed Tuberculosis in Virginia, USA. Tuberc Res Treat 2013;2013:129723.

41. Dooley KE, Chaisson RE. Tuberculosis and diabetes mellitus: convergence of two epidemics. Lancet Infect Dis 2009;9:737-46.

42. Matteelli A, Roggi A, Carvalho AC. Extensively drugresistant tuberculosis: epidemiology and management. Clin Epidemiol 2014;6:111-8.

43. Heysell SK, Moore JL, Keller SJ, et al. Therapeutic drug monitoring for slow response to tuberculosis treatment in a state control program, Virginia, USA. Emerg Infect Dis 2010;16:1546-53.

44. Gwilt PR, Nahhas RR, Tracewell WG. The effects of diabetes mellitus on pharmacokinetics and pharmacodynamics in humans. Clin Pharmacokinet 1991;20:477-90.

45. Liu Q, Li W, Xue M, et al. Diabetes mellitus and the risk of multidrug resistant tuberculosis: a meta-analysis. Sci Rep 2017;7:1090.

46. Frieden TR, Sterling T, Pablos-Mendez A, et al.The emergence of drug-resistant tuberculosis in New York City. N Engl J Med 1993;328:521-6.

47. Tsao TC, Chiou W, Lin H, et al. Change in demographic picture and increase of drug resistance in pulmonary tuberculosis in a 10-year interval in Taiwan. Infection 2002;30:75-80.

48. Tegegne BS, Mengesha MM, Teferra AA, et al. Association between diabetes mellitus and multi-drug-resistant tuberculosis: evidence from a systematic review and metaanalysis. Syst Rev 2018;7:161.

49. Gulland A. More cases of tuberculosis than previously thought, WHO reports. BMJ 2016;355:i5562.

50. Cheng J, Zhang H, Zhao YL, et al. Mutual Impact of Diabetes Mellitus and Tuberculosis in China. Biomed Environ Sci 2017;30:384-9.

51. Djuretic T, Herbert J, Drobniewski F, et al. Antibiotic resistant tuberculosis in the United Kingdom: 1993-1999. Thorax 2002;57:477-82.

52. Hayashi S, Chandramohan D. Risk of active tuberculosis among people with diabetes mellitus: systematic review and meta-analysis. Trop Med Int Health 2018;23:1058-70.

53. Jeon CY, Murray MB. Diabetes mellitus increases the risk of active tuberculosis: a systematic review of 13 observational studies. PLoS Med 2008;5:e152.

54. Leung CC, Lam TH, Chan WM, et al. Lower risk of 
tuberculosis in obesity. Arch Intern Med 2007;167:1297-304.

55. Kumar Nathella P, Babu S. Influence of diabetes mellitus on immunity to human tuberculosis. Immunology 2017;152:13-24.

56. Lopez-Lopez N, Martinez AGR, Garcia-Hernandez MH, et al. Type-2 diabetes alters the basal phenotype of human macrophages and diminishes their capacity to respond, internalise, and control Mycobacterium tuberculosis. Mem Inst Oswaldo Cruz 2018;113:e170326.

57. Baker MA, Harries AD, Jeon CY, et al. The impact of diabetes on tuberculosis treatment outcomes: a systematic review. BMC Med 2011;9:81.

58. Dooley KE, Tang T, Golub JE, et al. Impact of diabetes mellitus on treatment outcomes of patients with active tuberculosis. Am J Trop Med Hyg 2009;80:634-9.

59. Yew WW, Leung CC, Zhang Y. Oxidative stress and TB outcomes in patients with diabetes mellitus? J Antimicrob

Cite this article as: Huang D, Wang Y, Wang Y, Liang Z. The impact of diabetes mellitus on drug resistance in patients with newly diagnosed tuberculosis: a systematic review and metaanalysis. Ann Palliat Med 2020;9(2):152-162. doi: 10.21037/ apm.2020.02.16
Chemother 2017;72:1552-5.

60. Samuels JP, Sood A, Campbell JR, et al. Comorbidities and treatment outcomes in multidrug resistant tuberculosis: a systematic review and meta-analysis. Sci Rep 2018;8:4980.

61. Dos Santos Feltrin AF, Vendramini SH, Neto FC, et al. Death in patients with tuberculosis and diabetes: Associated factors. Diabetes Res Clin Pract 2016;120:111-6.

62. Shewade HD, Jeyashree K, Mahajan P, et al. Effect of glycemic control and type of diabetes treatment on unsuccessful TB treatment outcomes among people with TB-Diabetes: A systematic review. PLoS One 2017;12:e0186697.

63. Kossiǔ IE, Karachunskii MA, Kaminskaia GO, et al. Pulmonary tuberculosis in patients with different types of diabetes mellitus. Probl Tuberk 2002;(5):21-4. 